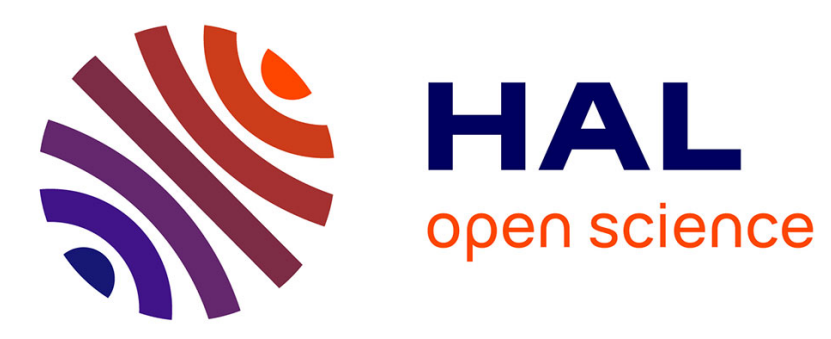

\title{
Responsibility Accounting with a Privately Informed Agent
}

François Larmande, Jean-Pierre Ponssard

\section{To cite this version:}

François Larmande, Jean-Pierre Ponssard. Responsibility Accounting with a Privately Informed Agent. 2007. hal-00243068

\section{HAL Id: hal-00243068 https://hal.science/hal-00243068}

Preprint submitted on 6 Feb 2008

HAL is a multi-disciplinary open access archive for the deposit and dissemination of scientific research documents, whether they are published or not. The documents may come from teaching and research institutions in France or abroad, or from public or private research centers.
L'archive ouverte pluridisciplinaire HAL, est destinée au dépôt et à la diffusion de documents scientifiques de niveau recherche, publiés ou non, émanant des établissements d'enseignement et de recherche français ou étrangers, des laboratoires publics ou privés. 


\section{ECOLE POLYTECHNIQUE}

CENTRE NATIONAL DE LA RECHERCHE SCIENTIFIQUE

\section{Responsibility Accounting with a Privately Informed} Agent

François Larmande

Jean-Pierre Ponssard

September 2007

Cahier n 2007-22

\section{LABORATOIRE D'ECONOMETRIE}

1rue Descartes F-75005 Paris

(33) 155558215

http://ceco.polytechnique.fr/

mailto:lyza.racon@shs.poly.polytechnique.fr 


\title{
Responsibility Accounting with a Privately Informed Agent ${ }^{1}$
}

\author{
François Larmande ${ }^{2}$ \\ Jean-Pierre Ponssard ${ }^{3}$
}

September 2007

Cahier n 2007-22

\section{Résumé:}

Abstract: The application of the controllability principle to eliminate external factors remains problematic in practice. A key issue concerns the fact that the manager may get some private pre-decision signal about this external environment and may use this information to mitigate or amplify its impact. The question is analyzed in a multitask agency model in which the agent has a

limited liability, thus earns rents and is not indifferent to the task performed. Under certain conditions, it is proved that the optimal contract only selectively eliminates the impact of the environment. It does neutralize it if the agent reports a signal of low impact of the environment but does not filter it out if the agent reports a signal of high impact.

\section{Mots clés :}

Key Words : controllability principle, responsibility accounting, multitask incentives with limited liability and private information

Classification JEL: $\quad$ D82, J33, M41

\footnotetext{
${ }^{1}$ We would like to thank Richard Lambert and other participants at the VIIth Workshop on Accounting and Economics (Bergen, 2006) for their comments on an early draft. Partial financial support from the Ecole polytechnique chair for Business Economics is gratefully acknowledged.

${ }^{2}$ EM-Lyon business school, larmande@em-lyon.com

${ }^{3}$ Ecole Polytechnique, Paris. jean-pierre.ponssard@polytechnique.edu
} 


\section{Introduction}

Should a manager be held accountable for observable and exogenous factors like the price of oil or foreign exchange rates? There is no consensus in the literature, even among proponents of agency theory. On the one hand Roberts (2004) recommends to follow the example of British Petroleum which removes the impact of the oil price from the performance measure of its managers. For him, it is a direct consequence of the "informativeness principle". By shielding the agent from this external factor, one can improve the power of incentives because there is less "noise" in the performance measure. On the other hand, Lambert (2001) develops the idea that even if the oil price is exogenous, one may still want the executive to attempt to forecast what oil prices will be and to design a strategy that corresponds to this forecast. Following this idea, Jensen and Murphy (2004) recommend to keep the external factor in the performance measure otherwise, they argue, managers would have no incentive to react to the environment if they can.

To investigate this issue we develop a multi-task agency model in which a principal (he) delegates to an agent (she) the management of a firm. It has the following properties. First the profit is impacted by an exogenous stochastic factor called the environment, the oil price for instance, which may have a high or a low impact on profit and which is publicly observed ex-post. Second there are two tasks: a "business as usual" task unrelated to the environment and a "react to the environment" task. The environment modifies the relative productivity of these tasks: it is worthwhile to "react to the environment" only if one forecasts it will have a high impact. Third, the manager receives a private but noisy signal of the ex-post realization of the environment before choosing her action. Thus, she knows better than the principal on which task it is optimal to work. Fourth, the agent has a limited liability (but is risk neutral). She therefore earns rents. We suppose that the "react to the environment" task, harder to monitor, delivers a higher rent. On top of moral hazard (efforts are not observable), the principal faces an adverse selection issue: all other things being equal, the agent will prefer to work on the "react to the environment" task. Thus, if the principal naively asks her to reveal her information, she will always report that the signal predicts 
a high impact of the environment.

We do not restrict in anyway the contract that the principal can offer. In particular communication is possible during the game, all observable variables can be used and a menu of contracts offered. The optimal contract depends on the quality of the private signal that is, how informative the signal is about the ex-post realization of the environment.

When its quality is good or intermediate, it is always possible to implement the congruent action: the agent will react to the environment if and only if she forecasts a high impact. The principal offers a menu of contracts. If the manager reports a low impact signal, the principal asks her to work on the "business as usual" task and the impact of the environment is completely filtered out. If the high impact signal is reported, the principal asked her to "react to the environment". But, in order to discipline her report, she is jointly responsible for the result of the task and the actual realization of the environment: if the "react to the environment" task is successful but the environment has actually a low impact she will not be rewarded. In this case, the principal uses a selective variance analysis: make the agent liable to the environment when she forecasts it will have a high impact and totally neutralized it otherwise.

When the quality of the signal is only intermediate (and not good), this "joint responsibility" is not enough: the principal also needs to increase the bonus of the low impact contract to induce a truthful report of the manager. This bonus increases as the quality worsens. When the quality is bad, it would become too costly to motivate the agent to perform the congruent action. A pooling contract is then optimal: the principal always asks the agent to work on the "business as usual" task and the impact of the environment is always filtered out. The agent will never react to it, even when she knows it would have been optimal to do so.

Our model shows that the existence and the quality of the private pre-decision information detained by the manager are key elements in the application of the controllability principle for external factors. To enlighten the results, it is proved that, were this pre-decision information public, it would be always possible to neutralize the impact of the environment without altering incentives to perform the congruent action. 
This paper extends the study of multi-task incentives, introduced by Holmström and Milgrom (1991) and Feltham and Xie (1994), with the design of a limited liability model. This offers a tractable framework to study the consequences of the private information of the agent regarding task productivity. We show in particular that the optimal weights placed on the different tasks are quite sensitive to the level of informational asymmetry: they are neither monotonic nor continuous with respect to the quality of the signal. This counter-intuitive result, as the one of Datar, Lambert and Kulp (2001) confirms the idea that the proper calibration of a multi-task incentive scheme is not a trivial issue.

The paper is organized as follows. Related literature is discussed in the next section. Section 3 introduces the model, then analyses the first best and the public signal case. Section 4 solves the general case. Section 5 discusses some additional issues: public but non contractible signal, value of information and optimal weights in multi-task incentives. Section 6 analyses the controllability principle in the light of our model and gives some concluding remarks. Proofs are in the appendix.

\section{Related literature}

This paper is related to four different streams of literature. The first one studies variance analysis as a motivational device following the seminal paper of Baiman and Demski (1980). As papers in this stream use a pure moral hazard framework, without any adverse selection issue, the informativeness principle applies, and it is always optimal to eliminate the impact of the environment through a variance analysis. Lambert (1985) introduces a friction: there is a direct cost to perform this variance investigation, you have to pay someone to perform this task. The issue is then to know when it is worth the cost: when results are very bad, very good or in line? This early literature does not point out the possible congruity drawback of variance analysis as we do when agents have some pre-decision information.

The second stream analyses the choice of performance measures as a trade-off between congruity (of the second best action the principal wants to induce compared to the first best) and controllability (incentive cost). This is a more general 
question that includes the traditional one: power of incentives versus agency cost. The multi-tasking modelling of Feltham and Xie (1994) allows to have a better understanding of this trade-off. In particular the principal may prefer to add a less congruent measure, like for instance annual accounting profit compared to stock price, because controllability improves, even if that distorts the choice of the agent towards the short term. In our paper we introduce two new ideas. First, congruity may not be definable ex-ante: it is stochastic because it depends on the realization of the signal. Second, the agent may know better than the principal what is the congruent action.

The third stream investigates the relation between noise and incentive strength. The Linear-Exponential-Normal model (LEN) introduced by Holmström and Milgrom (1987) implies a negative relationship. Yet, empirically, there is no clear relation. Prendergast (2002) argues that when noise increases, delegation may increase and so incentive strength too in a LEN framework. Here, like in Baker and Jorgensen (2003), we argue that environmental factors are not completely external because the agent can mitigate their impact. "Noise" may be kept in the performance measure to induce an optimal allocation of efforts across tasks. However, the criterion to decide to keep the noise or remove it is not the volatility of the noise, as in Baker and Jorgensen (2003), but the quality of the signal received by the agent before choosing her action.

Finally, from a technical point of view, there is a fourth stream of literature, the one that studies agency relationship with both moral hazard and adverse selection, because the agent has some private pre-decision information. It is a huge literature, and there is not a lot of general results. For instance, Christensen (1981) shows that the value of this private pre-decision information may be positive or negative for the principal. Here this value is always positive but we want to address another issue, the shape of the optimal contract: when and why to include the ex-post realization of the environment in the performance measure. 


\section{The Model}

A principal (he) hires a manager (she) to run a project, for instance a business unit. Both are risk neutral but the manager has a limited liability: in all states of the world, her wage must be positive.

The profit function of the project is defined as:

$$
\pi=A+\Theta\left(B-b_{0}\right)
$$

$A$ and $B$ are two random variables whose probability distributions depend on an effort made by the manager. They will be called task $A$ and task $B$. $\Theta$ represents the environment: a random variable whose probability distribution is not influenced by the agent. $b_{0}$ is a constant.

The fact that the environment comes in a multiplicative way, $\Theta . B$, is a key element of the model: the environment affects the relative productivity of tasks, thus impacts the optimal action to undertake. $\Theta$ may take two values 0 or 1 . If $\Theta=0$, we are in the "business as usual" case : $\pi=A$ and there is no point to work on task $B$. $A$ may thus be considered as the "business as usual" task. If $\Theta=1$, then the profit is negatively affected by the environment $\left(B-b_{0}\right.$ is assumed to be always negative). But it is possible to mitigate this impact by working on task $B$. For instance, if $\Theta$ represents the price of oil and if the project uses it as an input, then a successful task $B$ allows to reduce the quantity of oil used in the production process. As we will see below, the model is calibrated to ensure that it is worthwhile to work on task $B$ only if one forecasts that $\Theta$ will be equal to 1 .

The possible outcome of task $A$ (resp. $B$ ) is $\left\{0, x_{a}\right\}$ with $x_{a}>0$ (resp. $\left\{0, x_{b}\right\}$ with $x_{b}>0$ and $x_{b}<b_{0}$ ). The manager can exert an effort $e_{a}$ (resp. $e_{b}$ ) and incurs a private $\operatorname{cost} C_{a}$ (resp. $C_{b}$ ) or no effort at no cost. The outcome $x_{a}$ on $A$ (resp. $x_{b}$ on $B$ ) is obtained with probability $p_{a}$ (resp. $p_{b}$ ) if no effort is made on this task and with probability $p_{a}+e_{a}$ (resp. $p_{b}+e_{b}$ ) in case an effort $e_{a}$ (resp. $e_{b}$ ) is made. It is assumed at all times that $0 \leq p_{a}+e_{a} \leq 1$ and $0 \leq p_{b}+e_{b} \leq 1$. The following table summarizes these data: 


\begin{tabular}{|c|c|c|c|}
\hline task $I=A$ or $B$ & cost of effort & Prob $\left(I=x_{i}\right)$ & Prob $(I=0)$ \\
\hline no effort & 0 & $p_{i}$ & $1-p_{i}$ \\
\hline effort $e_{i}$ & $C_{i}$ & $p_{i}+e_{i}$ & $1-\left(p_{i}+e_{i}\right)$ \\
\hline
\end{tabular}

Due to a time constraint, the manager can work at most on one task: she must choose between an effort on task $A$, an effort on task $B$ or no effort at all.

Denote $\theta_{0}$ the ex-ante probability that $\Theta=1$. The agent receives a private signal of $\Theta$ before choosing her effort. This signal is the conditional probability that $\Theta=1$, denoted $\theta_{1}$. More precisely, $\theta_{1}$ may take two values $\theta_{H}$ and $\theta_{L}$ with $\theta_{L}<\theta_{0}<\theta_{H}$. It will be convenient to define $\lambda_{H}=\operatorname{Prob}\left(\theta_{1}=\theta_{H}\right)=\left(\theta_{0}-\right.$ $\left.\theta_{L}\right) /\left(\theta_{H}-\theta_{L}\right)$ and $\lambda_{L}=\operatorname{Prob}\left(\theta_{1}=\theta_{L}\right)=\left(\theta_{H}-\theta_{0}\right) /\left(\theta_{H}-\theta_{L}\right)$. By construction we have $\lambda_{H} \theta_{H}+\lambda_{L} \theta_{L}=\theta_{0}$

The realization of the environment $\Theta$ is public ex-post. But some asymmetry of information remains even after the observation of the environment. As the signal received ex-ante by the agent is not perfect, the principal does not know ex-post what the agent knew ex-ante. The model may exhibit therefore adverse selection on top of moral hazard. All variables $A, B$ and $\Theta$ are contractible. The principal can offer a menu of contracts to the agent in order to induce her to reveal her private information $\theta_{1}$.

Here is the timing of the game:

1. the principal proposes the bonus scheme which may involve a menu of contracts,

2. the manager gets a private signal $\theta_{1}$ of the environment,

3. if a menu of contracts has been offered, the manager selects the contract in the menu,

4. the manager makes her effort selectively on one task, either task $A$ or task $B$ (or no effort),

5. the outcomes of both tasks are publicly observed as well as the environment. Manager is paid. 
For all $\theta \in[0,1]$, let $\pi_{0}(\theta)=p_{a} x_{a}+\theta p_{b} x_{b}-\theta b_{0}$ be the expected profit of the principal if no effort is implemented and if the probability that $\Theta=1$ is $\theta$.

We are going to derive the first best. We will assume that:

$$
\theta_{H} e_{b} x_{b}-C_{b}>e_{a} x_{a}-C_{a}>\theta_{0} e_{b} x_{b}-C_{b}
$$

and that $e_{a} x_{a}-C_{a} \geq 0$.

The principal has the choice to work on task $A$, to work on task $B$ or not to work at all. If $\theta_{1}=\theta_{H}$, then the "incremental" expected profit (that is on top of $\left.\pi_{0}\left(\theta_{H}\right)\right)$ associated with an effort on task $A$ is $e_{a} x_{a}-C_{a}$ : the probability to obtain $x_{a}$ increases by $e_{a}$ but the principal incurs a cost $C_{a}$. If $\theta_{1}=\theta_{H}$ still, the incremental expected profit associated with an effort on task $B$ is $\theta_{H} e_{b} x_{b}-C_{b}$; as $\theta_{H} e_{b} x_{b}-C_{b}>e_{a} x_{a}-C_{a} \geq 0$, it is optimal for the principal if $\theta_{1}=\theta_{H}$ to work on task $B$. It is easy to see that if $\theta_{1}=\theta_{L}$, the reverse is true: the principal is better off to work on task $A$. Because, as $\theta_{0}>\theta_{L}$, assumption (??) gives $e_{a} x_{a}-C_{a}>\theta_{L} e_{b} x_{b}-C_{b}$. The following proposition sums up these results.

Lemma 1 The first best corresponds to the following situation:

- if $\theta_{1}=\theta_{L}$, the principal will choose to work on task $A$;

- if $\theta_{1}=\theta_{H}$, the principal will choose to work on task $B$.

Consider now the public signal case: the pre-decision signal is also observed by the principal. Since the agent has a limited liability, a compensation scheme $S$ takes the form of a bonus $\omega_{a} \geq 0$ in case task $A$ is successful, $A=x_{a}$, and no bonus in case it fails, $A=0$, and likewise a bonus on task $B, \omega_{b} \geq 0$, if $B=x_{b}$ and no bonus if $B=0$.

As the agent has only one unit of work, if the principal wants to induce $e_{a}$ (resp. $e_{b}$ ) there is no point to put a bonus on task $B$ (resp. $A$ ). Thus, $\omega_{a} \omega_{b}=0$.

The program of the principal can be broken down in two steps. First to compute the minimal bonus $\omega_{a}$ (resp. $\omega_{b}$ ) necessary to have effort $e_{a}$ (resp. $e_{b}$ ) 
implemented. Second to compare his incremental payoff for each action he can induce $\left(e_{a}, e_{b}\right.$ and 0$)$.

Consider first the implementation of $e_{a}$. The incentive constraint faced by the agent is:

$$
\left(p_{a}+e_{a}\right) \omega_{a}-C_{a} \geq p_{a} \omega_{a}
$$

The minimum bonus to implement $e_{a}$ is $\omega_{a}^{*}=C_{a} / e_{a}$. His expected payoff is $\pi_{a}(\theta)=e_{a} x_{a}-C_{a}-C_{a} p_{a} / e_{a}+\pi_{0}(\theta)$.

Likewise, the minimum bonus to implement $e_{b}$ is $\omega_{b}^{*}=C_{b} / e_{b}$ and the expected payoff of the principal is $\pi_{b}(\theta)=\theta e_{b} x_{b}-C_{b}-C_{b} p_{b} / e_{b}+\pi_{0}(\theta)$

Denote $\Delta \pi_{a}=e_{a} x_{a}-C_{a}-C_{a} p_{a} / e_{a}$ and $\Delta \pi_{b}(\theta)=\theta e_{b} x_{b}-C_{b}-C_{b} p_{b} / e_{b} . \Delta \pi_{a}$ represents for the principal the incremental expected profit associated with the implementation of $e_{a} . \Delta \pi_{b}(\theta)$ represents the corresponding incremental expected profit associated with task $B$ and the realization $\theta$ of the environment.

Compared to the first best, there is one extra term in the principal's payoff: $-C_{a} p_{a} / e_{a}$ or $-C_{b} p_{b} / e_{b}$ which is the rent given to the agent. This agency cost is increasing in $p_{i} / e_{i}$. This ratio $p_{i} / e_{i}$ can be seen as the "signal-to-noise ratio": the agency cost is lower when the effort of the agent, $e_{i}$, has a larger impact on the probability of success, and it increases with the "noise" $p_{i}$. In the traditional risk-averse agent model, the agency cost is also increasing in the "signal-to-noise ratio". But this agency cost is not a rent paid to the agent but a risk premium to be given to the agent to make her partially bear the risk.

It will be assumed that task $B$ is harder to monitor than task $A$ that is, the rent associated with task $B$ is higher than the one associated with task $A$ :

$$
p_{a} C_{a} / e_{a}<p_{b} C_{b} / e_{b}
$$

The second step in the principal's program is to select the action that gives the higher incremental payoff. We will assume that: 


$$
\begin{gathered}
\Delta \pi_{b}\left(\theta_{H}\right)>\Delta \pi_{a}>\Delta \pi_{b}\left(\theta_{0}\right) \\
\Delta \pi_{a}>0
\end{gathered}
$$

Assumption (??) ensures that action $e_{a}$ always dominates no effort. Since $\Delta \pi_{b}\left(\theta_{0}\right)>\Delta \pi_{b}\left(\theta_{L}\right)$, assumption (??) implies that $\Delta \pi_{b}\left(\theta_{H}\right)>\Delta \pi_{a}>\Delta \pi_{b}\left(\theta_{L}\right)$. As in the first best case, it is optimal in the public signal case to implement action $e_{a}$ in case $\theta_{1}=\theta_{L}$ while action $e_{b}$ should be implemented in case $\theta_{1}=\theta_{H}$.

Lemma 2 If the pre-decision signal received by the manager is public, the optimal contract is such that:

- if $\theta_{1}=\theta_{L}$, the principal asks the agent to make an effort $e_{a}$; a bonus $\omega_{a}^{*}=$ $C_{a} / e_{a}$ is paid if $A=x_{a}$ and no bonus is offered on task $B$.

- if $\theta_{1}=\theta_{H}$, the principal asks the agent to make an effort $e_{b}$; a bonus $\omega_{b}^{*}=$ $C_{b} / e_{b}$ is paid if $B=x_{b}$ and no bonus is offered on task $A$.

When the signal is public, there is no asymmetry of information between the principal and the manager regarding the optimal task to undertake. It is possible to eliminate the impact of environment without altering the congruity of the performance measure.

finally, since assumption (??) gives $\Delta \pi_{a}>\Delta \pi_{b}\left(\theta_{0}\right)$, if the signal were not informative at all, $\theta_{H}=\theta_{0}=\theta_{L}$, the principal would be better off to implement action $e_{a}$.

\section{The optimal contract with a privately informed agent}

We suppose now that the signal received by the manager is private. The principal has to deal with both moral hazard and adverse selection. Consider the following naive contract: the principal asks the manager to reveal her signal and then offers the contract of lemma (??). Such a scheme is easy to implement. It may be seen 
as providing different weights on the two tasks, i.e. $\left(\omega_{a}^{*}, \omega_{b}^{*}\right)$, while eliminating the impact of the environment through a standard variance analysis, which is feasible since the value of $\Theta$ is known ex-post. The trouble with this scheme is that, because of the asymmetry of information, the manager is encouraged to cheat. As $p_{b} C_{b} / e_{b}>p_{a} C_{a} / e_{a}$, her expected rent is higher if the principal asks her to implement task $B$. The expected incremental profit for the principal decreases to $\Delta \pi_{b}\left(\theta_{0}\right)$ since task $B$ is implemented any way. As $\Delta \pi_{b}\left(\theta_{0}\right)<\Delta \pi_{a}$ (assumption (??)), the principal would have been better off to ignore what the manager says and always ask to implement task $A$.

To solve this problem, the principal has two levers. The first one is to use his information about the ex-post realization of the environment to discipline the report made by the agent: when she announces that the signal forecasts a high impact of the environment, give the bonus in case task $B$ is successful but only if this task is worthwhile that is, $\Theta=1$. This means that the bonus $\omega_{b}$ will increase since it is obtained with a lower probability. The second lever is to increase the rent associated with task $A$. The following section makes this route precise.

Using the revelation principle one may conduct the analysis by comparing the principal payoff between the optimal (direct and truthful) revealing contract and the optimal pooling contract. A (direct and truthful) revealing contract determines the bonus of the agent $\omega_{i}$ for each task $I=A$ and $B$, depending on the signal that she announces, to be denoted $\widehat{\theta}_{1}$, and on the outcomes of the random variables $I$ and $\Theta$. Denote $\omega_{i}\left(\widehat{\theta}_{1}, I, \Theta\right)$ the corresponding bonus. There are sixteen bonuses to be determined. The situation is quite degenerated and there are many ways to implement the same outcome.

One key element to find the optimal contract will be the quality of the signal $\theta_{1}$. Define the following parameters:

$$
\begin{gathered}
\delta\left(\theta_{1}\right)=\left(p_{b} C_{b} / e_{b}\right) * \theta_{L} / \theta_{H}-p_{a} C_{a} / e_{a} \\
s\left(\theta_{1}\right)=\Delta \pi_{b}\left(\theta_{H}\right)-\Delta \pi_{a}-\delta\left(\theta_{1}\right) \lambda_{L} / \lambda_{H}
\end{gathered}
$$

We will see below the exact meaning of those two parameters. For the moment, let us remark that on the one hand, when $\theta_{L} / \theta_{H}$ is close enough to 1 that is, 
when the signal is not very informative, $\delta\left(\theta_{1}\right)>0$ (because of assumption (??): $\left.p_{a} C_{a} / e_{a}<p_{b} C_{b} / e_{b}\right)$. On the other hand, when $\theta_{L} / \theta_{H}$ is close to zero, $\delta\left(\theta_{1}\right)<0$.

As $\Delta \pi_{b}\left(\theta_{H}\right)>\Delta \pi_{a}$ (assumption (??)), $\delta\left(\theta_{1}\right)<0$ implies that $s\left(\theta_{1}\right)>0$. But the reverse is not true: if $\delta\left(\theta_{1}\right)>0$ but not too far from $0, s\left(\theta_{1}\right)$ remains positive.

Definition 3 The quality of the signal is said to be:

- good when $\theta_{H} / \theta_{L}$ is large enough, so that $\delta\left(\theta_{1}\right)<0$ and $s\left(\theta_{1}\right)>0$,

- bad when $\theta_{H} / \theta_{L}$ is close enough to 1 , so that $\delta\left(\theta_{1}\right)>0$ and $s\left(\theta_{1}\right)<0$,

- intermediate when $\delta\left(\theta_{1}\right)>0$ and $s\left(\theta_{1}\right)>0$

The quality of the signal is closely related to the notion of informativeness defined by Blackwell (1953). When $\theta_{H} / \theta_{L}$ increases, the signal becomes more informative and, all other things being equal, the quality of the signal also increases.

The shape of the optimal contact depends on the quality of the signal. For an easy reading, the optimal contracts are set out in three different propositions, the intermediate case, the most interesting one, first. Proofs of the three propositions are in the appendix.

Proposition 4 If the quality of the signal is intermediate, the principal offers a menu of contracts:

- if $\widehat{\theta}_{1}=\theta_{L}$, the principal asks the manager to make an effort $e_{a}$ a bonus $\omega_{a}=C_{a} / e_{a}+\delta\left(\theta_{1}\right) /\left(p_{a}+e_{a}\right)$ is paid $A$ iff $A=x_{a}$ and no bonus is offered on task $B$

- if $\widehat{\theta}_{1}=\theta_{H}$, the principal asks the manager to make an effort $e_{b}$ a bonus $\omega_{b}=C_{b} / e_{b} \theta_{H}$ is paid iff $\Theta=1$ and $B=x_{b}$ and no bonus is offered on task $A$.

In this case, the optimal contract is a separating one: the principal will tell the agent to work on either task, depending on the signal reported. If the signal 
reported is $\theta_{H}$, then the agent is responsible for the joint result $\Theta B$, that is for the task $B$ but also for $\Theta$. The observation of $\Theta$ ex-post is used to discipline the agent: she may be punished if the realization of $\Theta$ does not correspond to the signal reported ${ }^{1}$. If the signal reported is $\theta_{L}$ then the manager is only responsible for the "business as usual" $A$-task. In that case, both $\Theta$ and the task $B$ are considered to be outside the control of the manager and filtered out of the performance measure.

Compared to the public signal case, the bonus $\omega_{a}$ has to increase by $\delta\left(\theta_{1}\right) /\left(p_{a}+\right.$ $\left.e_{a}\right)$ in order to fulfill the revelation constraint. Otherwise, the agent would always announce $\widehat{\theta}_{1}=\theta_{H}$ even if she observed $\theta_{L}$; the agent would gamble that $\Theta=1$ despite the opposite signal $\theta_{L}$. $\theta_{L}$ is precisely the probability that $\Theta=1$ when $\theta_{1}=\theta_{L} . \delta\left(\theta_{1}\right)$ represents the opportunity payoff of the manager associated with this gamble strategy. When the quality is intermediate (and bad) this payoff is indeed positive. As $\delta\left(\theta_{1}\right)$ increases when $\theta_{L} / \theta_{H}$ increases, the agency cost increases when the quality worsens.

It remains to see that the principal is better off with this optimal revealing contract rather than with the optimal pooling contract. As $\Delta \pi_{a} \geq \Delta \pi_{b}\left(\theta_{0}\right)$, in this optimal pooling contract the principal asks the manager to work on task $A$. The incremental expected gross profit of the revealing contract in case $\hat{\theta}_{1}=\theta_{H}$ is $\left(\Delta \pi_{b}\left(\theta_{H}\right)-\Delta \pi_{a}\right)$ while the incremental cost in case $\hat{\theta}_{1}=\theta_{L}$ is $\delta\left(\theta_{1}\right)$. Since $\lambda_{H}\left(\Delta \pi_{b}\left(\theta_{H}\right)-\Delta \pi_{a}\right)-\lambda_{L} \delta\left(\theta_{1}\right)=\lambda_{H} s\left(\theta_{1}\right)$ the optimality of the revealing contract over the pooling one amounts to $s\left(\theta_{1}\right) \geq 0$, which is indeed in the case when the quality of the signal is intermediate.

The worsening of the signal quality has two effects which go in the same direction. First the incremental expected gross profit, $\Delta \pi_{b}\left(\theta_{H}\right)-\Delta \pi_{a}$, decreases: as the informativeness of the signal worsens, the action undertaken (based on the signal) will more often appear not to be optimal ex-post (once the exact realization of the environment is known). Second, the incentive cost $\delta\left(\theta_{1}\right)$ increases. It

\footnotetext{
${ }^{1}$ As the agent is risk neutral in our model, there is no loss for the principal to include the environment in the performance measure. In a more general framework with both limited liability and risk aversion, the agent should be compensated for this additional risk.
} 
becomes more difficult for the principal to infer from the observation of the environment ex-post the signal observed ex-ante by the agent. Thus the asymmetry of information increases.

When $s\left(\theta_{1}\right)<0$ that is, when the quality of the signal is bad, the principal is better off with the pooling contract: tell the agent to always work on task $A$, whatever the signal received. It would become too costly to induce the agent to reveal her information. The principal is then better off to give up congruity (which is to work on task $B$ if $\theta_{1}=\theta_{H}$ ) in order to decrease the agency cost. He performs a rough variance analysis and remove totally the environment from the performance measure.

Proposition 5 If the quality of the signal is bad, there is only one contract:

- the principal asks the manager to make an effort $e_{a}$ a bonus $\omega_{a}=C_{a} / e_{a}$ is paid $A$ iff $A=x_{a}$ and no bonus is offered on task $B$

It remains to investigate the good quality case. Then $\delta\left(\theta_{1}\right)<0$ : the payoff associated with the "gamble" strategy is negative, because the ex-post realization of the environment is too often aligned with the signal. Even with the minimum bonus $\omega_{a}=C_{a} / e_{a}$ on task $A$, the manager has no incentive to announce $\widehat{\theta}_{1}=\theta_{H}$ when she actually observed $\theta_{L}$.

Proposition 6 If the quality of the signal is good, the principal offers a menu of contracts:

- if $\widehat{\theta}_{1}=\theta_{L}$, the principal asks the manager to make an effort $e_{a}$ a bonus $\omega_{a}=C_{a} / e_{a}$ is paid $A$ iff $A=x_{a}$ and no bonus is offered on task $B$

- if $\widehat{\theta}_{1}=\theta_{H}$, the principal asks the manager to make an effort $e_{b}$ $a$ bonus $\omega_{b}=C_{b} / e_{b} \theta_{H}$ is paid iff $\Theta=1$ and $B=x_{b}$ and no bonus is offered on task $A$.

This contract is close to the public signal contract of lemma (??). The only difference is that when the manager works on $\operatorname{task} B$, she is liable for the joint 
result $\Theta B$. It is crucial for the principal to hold the manager responsible for $\Theta$. Suppose that $\Theta$ cannot be included in the performance measure. The principal could still induce the agent to use her knowledge, by increasing the rent associated with task $A$ until it became equal to the rent associated with task $B$. The manager would then be indifferent to the task performed (and as usual we suppose she would choose to perform the task preferred by the principal). But that would very be costly for the principal. Whatever the realization of the signal the manager would receive a rent equal to $p_{b} C_{b} / e_{b}$. In fact, it would be too costly to induce the agent to react to the environment. The principal would be better off to use a pooling contract and ask the manager to always work on task $A$.

The menus of contracts of the good and intermediate quality cases can be interpreted as an interactive process. The principal offers at the beginning of the period a bonus only on task $A$; the exact value of this bonus depends of the quality of the signal: $C_{a} / e_{a}+\delta\left(\theta_{1}\right) /\left(p_{a}+e_{a}\right)$ or $C_{a} / e_{a}$. In the middle of the period, the manager may tell the principal (when she observes $\theta_{1}=\theta_{H}$ ) that she has the feeling the environment will be high. Then the contract is modified: the principal asks the agent to work on task $B$ but the environment is included in the performance measure.

\section{Complements}

\subsection{Signal publicly observed ex-post but non-contractible}

Suppose that at the end of the game the principal observes the early signal of the agent but cannot contract upon it ex-ante. The principal could tell the agent to do her best (that is, to work on the task she thinks is the more valuable) and that she will be rewarded according to the value of the signal observed expost. But there is a possible hold-up from the principal. Suppose for instance that $\theta_{1}=\theta_{H}$, task $B$ is successful but task $A$ fails. Then the principal could say ex-post: I think you had observed $\theta_{1}=\theta_{L}$; you should therefore have worked on $\operatorname{task} A$. As task $A$ is not successful, you will earn no bonus. A similar argument 
applies if task $A$ is successful but task $B$ fails. Because of this possible hold-up, the optimal reaction of the agent to a "do your best" order is to never make any effort. Thus the principal has to commit ex-ante by offering the same menu of contracts as above. There is therefore no gain for the principal to know ex-post the early signal observed by the agent if it is not contractible ${ }^{2}$.

\subsection{Value of information}

If there were no signal at all, then $\theta_{L}=\theta_{H}=\theta_{0}$, and it is easy to show that the optimal no-signal contract would be the same as the pooling contract of proposition (??). As assumption (??) gives $e_{a} x_{a}-C_{a}>\theta_{0} e_{b} x_{b}-C_{b}$ even the first best would be to work on the "business as usual" task $A$. Thus the private information of the agent has a positive value both for the manager and for the principal. For the agent, because in the no-signal case the principal always asks to work on task $A$, which yields the minimum rent $\left(p_{a} C_{a} / e_{a}\right)$ she can obtain if she is privately informed. For the principal because if he offered the no-signal contract to a privately informed agent, the latter would always choose to work on task $A$ just like a non-informed agent.

Corollary 7 The information of the agent has a positive value both for the principal and the agent.

Figure (1) explains how to obtain the value of information for the principal. Figure (2) gives the value of information both for the agent and for the principal with respect to the quality of the signal ${ }^{3}$. We can see that their preferences regarding the quality of the signal are not aligned.

\footnotetext{
${ }^{2}$ In practice this possible hold-up is a real issue. It can be related to the hindsight effect pointed out by Merchant and Van der Stede (2003) and coined by Hastie and Hawkins (1990): evaluators with knowledge of the results tend to assume information about the pre-result circumstances that was not available to those being evaluated.

${ }^{3}$ The value of information for the agent is not directly her rent but the difference between her rent and the rent she would obtain in the no-signal case, $R_{a}=p_{a} C_{a} / e_{a}$. For instance in the good quality case the value of information for the agent is $\lambda_{L} R_{a}+\lambda_{H} R_{b}-R_{a}=\lambda_{H}\left(R_{b}-R_{a}\right)$
} 
Corollary 8 The principal values the signal according to its quality (good preferred to intermediate preferred to bad). The agent prefers a signal of intermediate quality, then good finally bad.

Incentives to have the agent gather information about the environment are not aligned. The principal prefers a very informative signal. The agent prefers a signal of intermediate quality, precisely to be just on the right of the bad/intermediate quality frontier. If the quality of the signal itself resulted from an effort or an action of the agent but were hard information ${ }^{4}$, it would be in her interest to generate an intermediate quality. Not too low to be allowed to work on task $B$, but not too high to keep an informational advantage over the principal.

\subsection{Optimal weights in multi-task incentives}

As in our model the agent has only one "unit of time" - she can work only on one task, there is no real weights in the performance measure, aggregating results of both tasks. However we can use the bonuses themselves as a proxy for the weights we would find in a more sophisticated model with the possibility to work on both tasks simultaneously. The following figure draws the evolution of optimal bonuses with respect to the quality of the signal.

\section{Insert figure 3 here}

At first, when the quality is bad, $\omega_{b}$ is equal to zero and $\omega_{a}>0$, but both remain constant as the quality increases. There is a discontinuity when the quality hits the bad-intermediate frontier: both $\omega_{a}$ and $\omega_{b}$ undergo a one-time increase then decrease as the quality increases. When the quality becomes good, the bonus $\omega_{a}$ remains constant (equal to its initial level) whereas $\omega_{b}$ continues to decrease.

\footnotetext{
${ }^{4}$ Which means that once the agent has revealed the quality, the principal can audit the truthfulness of this information.
} 
The weights are therefore neither monotonic nor continuous with respect to the quality of the signal. A similar point was made by Datar, Lambert and Kulp (2001) in the multi-task LEN model: the weight placed on a performance measure may decrease as the accuracy of this measure increases, contrary to the common sense. Both models suggest that the calibration of the compensation function over several performance measures, though solvable in theory, is quite sensitive to the parameters involved. They may explain the difficulties encountered in practice (see Ittner, Larcker and Meyer, 2003).

\section{Controllability principle and concluding remarks}

\subsection{Some limitations of the model}

As the model is intentionally simple, the reader may question the generality of the result. First, it seems possible to introduce different levels of efforts, more than one "unit of time" for the manager and a possible balanced distribution of efforts across tasks (possibility to work a little on task $A$ and little on task $B$ instead of only on task $A$ or on task $B$ ) without changing the main conclusions. Second, it would be worthwhile to investigate what happens if the rent associated with task $A$ is higher than the one associated with task $B$, and more generally if productivity of both tasks are unknown. One could also investigate how to shape incentives if the signal is not free but requires an effort of the agent to generate it as in Lambert (1986).

More importantly, the manager is assumed to be risk neutral in our model. There is therefore no cost to include the environment in the performance measure. What would happen if the manager were risk-averse? We can conjecture that our main result - to include the environment in the performance measure allows to decrease the incentive cost - remains valid, albeit only for higher values of the quality of the signal than in the risk neutral case. 


\subsection{A reformulation of the controllability principle}

The traditional presentation of the controllability principle stipulates that a manager should be evaluated only on what he or she can control. But, as Lambert (2001) points out, in that definition the meaning of "control" is not well specified. Antle and Demski (1988) show that this definition must be understood in a very broad sense. For instance, when a relative performance evaluation is used, there is something managers do not control in the performance measure: peer actions, peer luck... Yet it seems natural and fair to use such relative evaluation. The controllability principle should then be stated as: all information that can reduce the noise of the performance measure should be used to evaluate the manager. This is the informativeness principle of the agency theory (Holmström 1979, Gjesdal 1982).

Here, even this formulation is violated. The manager does not "control" the environment that is, her actions have no impact on the probability distribution of the environment. Yet in some circumstances it is optimal to keep this uncertainty in the performance measure in order to lower the incentive cost and to motivate the manager to use optimally her private information. We cannot neither state an "influenceability principle" like all noises which could trigger a reaction of the manager to mitigate or amplify its impact should remain in the performance measure. The criterion to decide whether to include an environmental factor or to exclude it is the quality of the signal. If the quality is too low, it would be too costly to induce the manager to perform the congruent action. Our result offers a rationale for the fact that the controllability principle is not always applied by firms for external economic factors, as documented by Merchant (1989) and recommended by Jensen and Murphy (2004). The next table summarizes our results concerning the application of the controllability principle and its impact on the implementation of the congruent action: 


\begin{tabular}{|c|c|c|c|c|}
\hline Signal & \multicolumn{3}{|c|}{ Private } & Public \\
\hline Quality of the signal & Bad & Intermediate & Good & - \\
\hline Controllability principle & Applied & Selectively applied & Applied \\
\hline Congruent action always implemented & No & \multicolumn{2}{|c|}{ Yes } \\
\hline
\end{tabular}

\subsection{Managerial relevance}

To enlighten the relevance of our model, consider a recent empirical discussion of compensation schemes in which controllability is at stake. Bertrand and Mullainathan (2001) argued that top managers are "rewarded for luck" rather than for effort. According to the authors, if only effort would be rewarded, the observed bonuses should not depend on external uncontrollable events such as the price of oil. Our model proves that a reward for luck may be the optimal response when top managers have private pre-decision information on these events and can react to it. To see that consider this slight variation of our model. Suppose that $b_{0}=0$ but $x_{b}$ remains positive. Now a high impact of the environment $(\Theta=1)$ is good news for the firm: the profit is positively affected by the environment. An effort on task $B$ allows now to take advantage of the environment. It is easy to see that all our results remain valid. The contracts of the good and intermediate quality cases exhibit a "reward for luck" shape: the bonus depends on $\Theta$ when the signal forecasts it will be favorable $\left(\theta_{1}=\theta_{H}\right)$, and does not depend on $\Theta$ when the signal forecasts it will not be favorable $\left(\theta_{1}=\theta_{L}\right)$.

\section{Appendix: proof of propositions ??, ?? and ??}

Recall that $\omega_{i}\left(\hat{\theta}_{1}, I, \Theta\right)$ is the bonus earned by the agent depending on what she announces, $\hat{\theta}_{1}$, and on the realization of the random variables $I$ and $\Theta$.

We are going to derive first the optimal revealing contracts. Then we will compare its outcome with the optimal pooling contract. 


\subsection{Revealing contracts}

According to the revelation principle we can restrict the search of optimal revealing contracts to direct truthful mechanisms.

If the agent observes $\theta_{H}$, then because of assumption (??), the principal wants to implement task $e_{b}$. There is no point to reward the agent if the task fails $(B=0)$. Thus $\omega_{b}\left(\theta_{H}, 0,1\right)=\omega_{b}\left(\theta_{H}, 0,0\right)=0$

The first incentive constraint is thus:

$$
e_{b}\left[\theta_{H} \omega_{b}\left(\theta_{H}, x_{b}, 1\right)+\left(1-\theta_{H}\right) \omega_{b}\left(\theta_{H}, x_{b}, 0\right)\right]-C_{b} \geq 0
$$

On the other hand, if the agent observes $\theta_{L}$, the principal wants to implement task $e_{a}$. Here also $\omega_{a}\left(\theta_{L}, 0,1\right)=\omega_{a}\left(\theta_{L}, 0,0\right)=0$

The second incentive constraint is therefore:

$$
e_{a}\left[\theta_{L} \omega_{a}\left(\theta_{L}, x_{a}, 1\right)+\left(1-\theta_{L}\right) \omega_{a}\left(\theta_{L}, x_{a}, 0\right)\right]-C_{a} \geq 0
$$

We are going now to find the revelation constraints. If the agent lies and announces $\theta_{L}$ when she really observes $\theta_{H}$, what will be her optimal action? An effort $e_{a}$ or no effort at all?

If she chooses no effort, her net payoff would be

$$
\begin{aligned}
& p_{a}\left[\theta_{H} \omega_{a}\left(\theta_{L}, x_{a}, 1\right)+\left(1-\theta_{H}\right) \omega_{a}\left(\theta_{L}, x_{a}, 0\right)\right] . \text { And if she chooses } e_{a}, \\
& \left(p_{a}+e_{a}\right)\left[\theta_{H} \omega_{a}\left(\theta_{L}, x_{a}, 1\right)+\left(1-\theta_{H}\right) \omega_{a}\left(\theta_{L}, x_{a}, 0\right)\right]-C_{a} .
\end{aligned}
$$

Let $G\left(\hat{\theta}=\theta_{L}, \theta_{1}=\theta_{H}\right)$ be the maximum of those two payoffs.

Likewise, if the agent lies and announces $\theta_{H}$ when she really observes $\theta_{L}$, then her net payoff would be:

$$
G\left(\hat{\theta}=\theta_{H}, \theta_{1}=\theta_{L}\right)=\max \left[\begin{array}{c}
p_{b}\left[\theta_{L} \omega_{b}\left(\theta_{H}, x_{b}, 1\right)+\left(1-\theta_{L}\right) \omega_{b}\left(\theta_{H}, x_{b}, 0\right)\right] ; \\
\left(p_{b}+e_{b}\right)\left[\theta_{L} \omega_{b}\left(\theta_{H}, x_{b}, 1\right)+\left(1-\theta_{L}\right) \omega_{b}\left(\theta_{H}, x_{b}, 0\right)\right]-C_{b}
\end{array}\right]
$$


The two revelation constraints are thus:

$$
\begin{aligned}
& \left(p_{b}+e_{b}\right)\left[\theta_{H} \omega_{b}\left(\theta_{H}, x_{b}, 1\right)+\left(1-\theta_{H}\right) \omega_{b}\left(\theta_{H}, x_{b}, 0\right)\right]-C_{b} \geq G\left(\hat{\theta}=\theta_{L}, \theta_{1}=\theta_{H}\right) \\
& \left(p_{a}+e_{a}\right)\left[\theta_{L} \omega_{a}\left(\theta_{L}, x_{a}, 1\right)+\left(1-\theta_{L}\right) \omega_{a}\left(\theta_{L}, x_{a}, 0\right)\right]-C_{a} \geq G\left(\hat{\theta}=\theta_{H}, \theta_{1}=\theta_{L}\right)
\end{aligned}
$$

The principal minimizes the expected wage bill under those four constraints, $(? ?),(? ?),(? ?)$ and (??).

It is always possible to set $\omega_{b}\left(\theta_{H}, x_{b}, 0\right)=0$. For a given level of expected wage in the state of the world $\theta_{1}=\theta_{H}$, that is for $\theta_{H} \omega_{b}\left(\theta_{H}, x_{b}, 1\right)+\left(1-\theta_{H}\right) \omega_{b}\left(\theta_{H}, x_{b}, 0\right)$ remaining constant, by setting $\omega_{b}\left(\theta_{H}, x_{b}, 0\right)=0$, the principal does not change (??) nor (??) but (??) becomes more slack, because $\theta_{H}>\theta_{L}$.

Hence (??) becomes

$$
\omega_{b}\left(\theta_{H}, x_{b}, 1\right) \geq C_{b} /\left(\theta_{H} e_{b}\right)
$$

$5^{\prime}(9)$

$$
G\left(\hat{\theta}=\theta_{H}, \theta_{1}=\theta_{L}\right)=\max \left[\begin{array}{c}
p_{b} \theta_{L} \omega_{b}\left(\theta_{H}, x_{b}, 1\right) \\
\left(p_{b}+e_{b}\right) \theta_{L} \omega_{b}\left(\theta_{H}, x_{b}, 1\right)-C_{b}
\end{array}\right]
$$

and (??) becomes:

$$
\left(p_{b}+e_{b}\right) \theta_{H} \omega_{b}\left(\theta_{H}, x_{b}, 1\right)-C_{b} \geq G\left(\hat{\theta}=\theta_{L}, \theta_{1}=\theta_{H}\right)
$$

$7^{\prime}(10)$

We are going now to suppose that (??) is saturated, that is to set

$\omega_{b}\left(\theta_{H}, x_{b}, 1\right)=C_{b} /\left(\theta_{H} e_{b}\right)$. We will show that 1$)$ this is always feasible and 2$)$ it is not possible for the principal to improve his welfare by setting $\omega_{b}\left(\theta_{H}, x_{b}, 1\right)>$ $C_{b} /\left(\theta_{H} e_{b}\right)$.

Then 
$e_{b} \theta_{L} \omega_{b}\left(\theta_{H}, x_{b}, 1\right)=e_{b} \theta_{L} C_{b} /\left(\theta_{H} e_{b}\right)=C_{b} \theta_{L} / \theta_{H}<C_{b}$

Hence

$G\left(\hat{\theta}=\theta_{H}, \theta_{1}=\theta_{L}\right)=p_{b} \theta_{L} \omega_{b}\left(\theta_{H}, x_{b}, 1\right)=p_{b} C_{b} / e_{b} *\left(\theta_{L} / \theta_{H}\right)$

Let $\omega_{a}=\theta_{L} \omega_{a}\left(\theta_{L}, x_{a}, 1\right)+\left(1-\theta_{L}\right) \omega_{a}\left(\theta_{L}, x_{a}, 0\right)$. The principal minimizes $\omega_{a}$ under the constraints (??) and (??) (provided that (??) is verified), which can be rewritten as:

$$
\begin{aligned}
e_{a} \omega_{a}-C_{a} & \geq 0 \\
\left(p_{a}+e_{a}\right) \omega_{a}-C_{a} & \geq p_{b} C_{b} / e_{b} *\left(\theta_{L} / \theta_{H}\right)
\end{aligned}
$$

Recall that $\delta\left(\theta_{1}\right)=\left(p_{b} C_{b} / e_{b}\right) * \theta_{L} / \theta_{H}-p_{a} C_{a} / e_{a}$. Hence $\left(p_{a}+e_{a}\right) \omega_{a}-C_{a} \geq$ $p_{b} C_{b} / e_{b} *\left(\theta_{L} / \theta_{H}\right) \Longleftrightarrow \omega_{a} \geq C_{a} / e_{a}+\delta\left(\theta_{1}\right) /\left(p_{a}+e_{a}\right)$

Thus $\omega_{a}=\max \left[C_{a} / e_{a} ; C_{a} / e_{a}+\delta\left(\theta_{1}\right) /\left(p_{a}+e_{a}\right)\right]$

The condition $C_{a} / e_{a} \geq C_{a} / e_{a}+\delta\left(\theta_{1}\right) /\left(p_{a}+e_{a}\right)$ is equivalent to $\delta\left(\theta_{1}\right) \leq 0$.

If $\delta\left(\theta_{1}\right) \leq 0,(? ?)$ is binding and $\omega_{a}=C_{a} / e_{a}$.

This situation is optimal for the principal because the two incentive constraints are binding (remember that the principal wants to minimize the expected wage bill which is a linear combination of the left hand sides of both incentive constraints).

if on the contrary $\delta\left(\theta_{1}\right) \geq 0,(? ?)$ is binding and $\omega_{a}=C_{a} / e_{a}+\delta\left(\theta_{1}\right) /\left(p_{a}+e_{a}\right)$

Can the principal improve his welfare by decreasing $\omega_{a}$ and increasing $\omega_{b}\left(\theta_{H}, x_{b}, 1\right)$ ? No because then the LHS of (??) would decrease and the RHS would increase, which is not possible because (??) is already binding.

It remains to see that (??) is verified in both cases. The principal has one degree of freedom left "inside" $\omega_{a}$ : the distribution between $\omega_{a}\left(\theta_{L}, x_{a}, 1\right)$ and $\omega_{a}\left(\theta_{L}, x_{a}, 0\right)$. We are going to show that by setting $\omega_{a}\left(\theta_{L}, x_{a}, 1\right)=\omega_{a}\left(\theta_{L}, x_{a}, 0\right)=$ $\omega_{a},(? ?)$ is verified. 


$$
\begin{aligned}
G\left(\hat{\theta}=\theta_{L}, \theta_{1}=\theta_{H}\right) & =\max \left(\begin{array}{c}
p_{a}\left[\theta_{H} \omega_{a}\left(\theta_{L}, x_{a}, 1\right)+\left(1-\theta_{H}\right) \omega_{a}\left(\theta_{L}, x_{a}, 0\right)\right] ; \\
\left(p_{a}+e_{a}\right)\left[\theta_{H} \omega_{a}\left(\theta_{L}, x_{a}, 1\right)+\left(1-\theta_{H}\right) \omega_{a}\left(\theta_{L}, x_{a}, 0\right)\right]-C_{a}
\end{array}\right) \\
& =\max \left[p_{a} \omega_{a} ;\left(p_{a}+e_{a}\right) \omega_{a}-C_{a}\right]
\end{aligned}
$$

As $\omega_{a}=\max \left[C_{a} / e_{a} ; C_{a} / e_{a}+\delta\left(\theta_{1}\right) /\left(p_{a}+e_{a}\right)\right]$, we have $\omega_{a} \geq C_{a} / e_{a}$, thus:

$$
G\left(\hat{\theta}=\theta_{L}, \theta_{1}=\theta_{H}\right)=\left(p_{a}+e_{a}\right) \omega_{a}-C_{a}
$$

The LHS of (??) is equal to $\left(p_{b}+e_{b}\right) C_{b} / e_{b}-C_{b}=p_{b} C_{b} / e_{b}$

If $\delta\left(\theta_{1}\right)<0, \omega_{a}=C_{a} / e_{a}$ and (??) is equivalent to $p_{b} C_{b} / e_{b} \geq p_{a} C_{a} / e_{a}$ which is true because of assumption (??).

In the other hand, if $\delta\left(\theta_{1}\right) \geq 0$, that means that (??) is binding, so $\left(p_{a}+e_{a}\right) \omega_{a}-$ $C_{a}=p_{b} \theta_{L} C_{b} /\left(\theta_{H} e_{b}\right)$, which is less than $p_{b} C_{b} / e_{b}$ because $\theta_{L} / \theta_{H}<1$, and (??) is verified.

\subsection{Comparison with the pooling contract}

We have finished to derive the optimal revealing contract. It remains to compare it to the optimal pooling contract.

Assumption (??) entails that the principal wants to implement $e_{a}$ in the pooling case. The agent will receive a wage $C_{a} / e_{a}$ in case of success. And the payoff of the principal in the pooling case is: $e_{a} x_{a}-C_{a}-p_{a} C_{a} / e_{a}$

Recall that $\lambda_{H}$ is the probability that $\theta=\theta_{H}: \lambda_{H}=\left(\theta-\theta_{L}\right) /\left(\theta_{H}-\theta_{L}\right)$

Therefore, $\left(1-\lambda_{H}\right) / \lambda_{H}=\left(\theta_{H}-\theta_{L}\right) /\left(\theta-\theta_{L}\right)-1=\left(\theta_{H}-\theta\right) /\left(\theta-\theta_{L}\right)$

The payoff of the principal with the optimal revealing contract is, if $\delta\left(\theta_{1}\right)<0$, $\left(1-\lambda_{H}\right)\left[e_{a} x_{a}-C_{a}-p_{a} C_{a} / e_{a}\right]+\lambda_{H}\left[\theta_{H} e_{b} x_{b}-C_{b}-p_{b} C_{b} / e_{b}\right]$ which is superior or equal to $e_{a} x_{a}-C_{a}-p_{a} C_{a} / e_{a}$ because of assumption (??).

If $\delta\left(\theta_{1}\right) \geq 0$, the payoff is $\left(1-\lambda_{H}\right)\left[e_{a} x_{a}-C_{a}-\delta\left(\theta_{1}\right)-p_{a} C_{a} / e_{a}\right]+\lambda_{H}\left[\theta_{H} e_{b} x_{b}-C_{b}-p_{b} C_{b} / e_{b}\right]$

because when $\delta\left(\theta_{1}\right) \geq 0,(? ?)$ is binding, and

$$
\left(p_{a}+e_{a}\right) \omega_{a}=C_{a}+p_{b} \theta_{L} C_{b} /\left(\theta_{H} e_{b}\right)=C_{a}+\delta\left(\theta_{1}\right)+p_{a} C_{a} / e_{a}
$$


The payoff is greater in the pooling case iff:

$\left(1-\lambda_{H}\right)\left[e_{a} x_{a}-C_{a}-\delta\left(\theta_{1}\right)-p_{a} C_{a} / e_{a}\right]+\lambda_{H}\left[\theta_{H} e_{b} x_{b}-C_{b}-p_{b} C_{b} / e_{b}\right] \leq e_{a} x_{a}-C_{a}-p_{a} C_{a} / e_{a}$ that is, iff:

$-\left[e_{a} x_{a}-C_{a}-p_{a} C_{a} / e_{a}\right]-\left(1-\lambda_{H}\right) \delta\left(\theta_{1}\right) / \lambda_{H}+\left[\theta_{H} e_{b} x_{b}-C_{b}-p_{b} C_{b} / e_{b}\right] \leq 0$

that is iff $s\left(\theta_{1}\right) \leq 0$.

The principal offers a revealing contract if and only if $s\left(\theta_{1}\right)>0$. This is the case when the quality of the signal is either good or intermediate. 